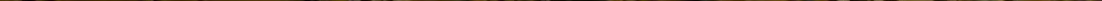







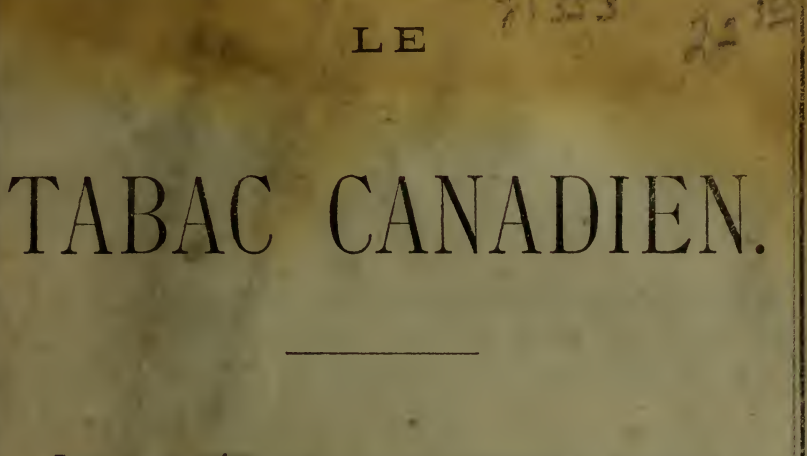

LOIS QUI RÉGISSENT LA CULTURE ET LA VENTE du Tabac Canadien.

Extraits du chapitre ro des Statuts du Canada, 1880.

\section{QUEBFO :}

- DE L'IMPRIMERIE DE L. J. BEMERS \& FRÈRE, Editeurs-Prop"iétaires du "Canadien." 

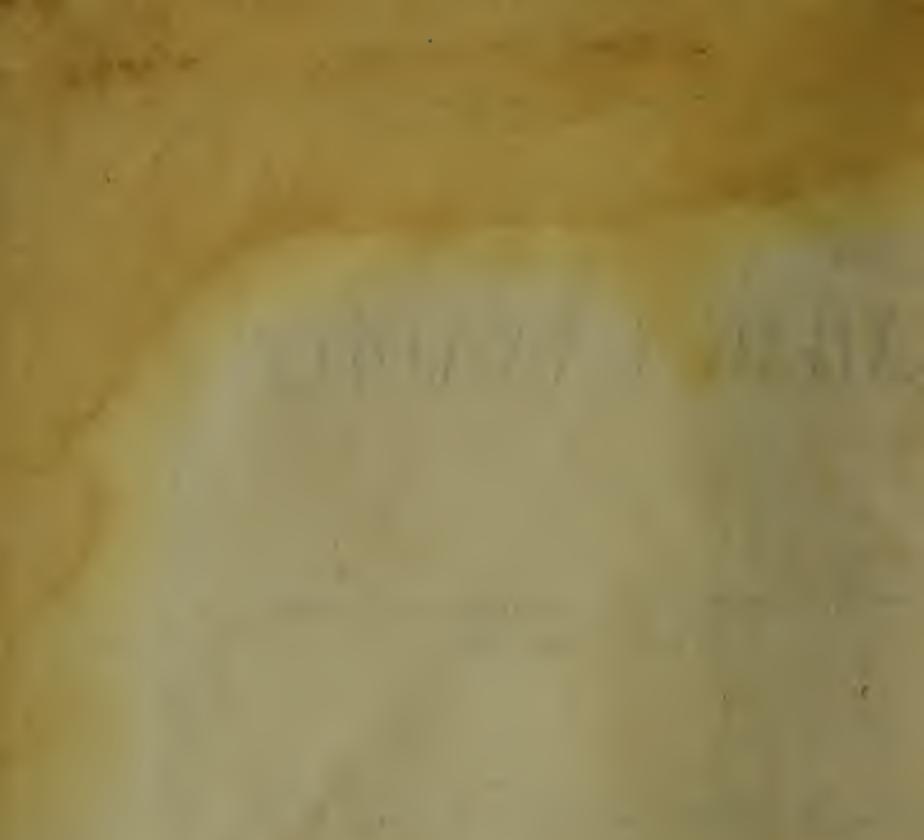

$-$

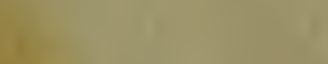

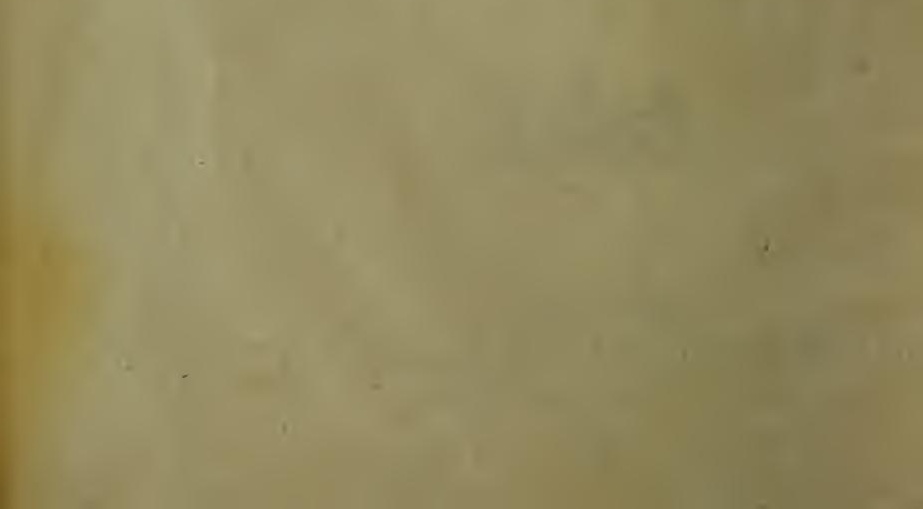




\section{PRÉFACE.}

Nnus offrons aux cultivateurs canadiens la petite brochure actuelle pour les mettre en posit on de comnaître bien et facilement la loi qui régit la culture et la fabrication du tabac.

Les réponses données aux questions posées sont puisétes dans le texte même de la loi.

Cette nouvelle loi a été faite pour encourager et protéger le cultivateur canadien dans la culture du tabac. C'est à chacuu d'en profiter, en s'y conformant fidèlement.

Nous avons cru utile de faire précéder ce petit catéchisme légal de quelques courtes observations sur la culture et la fabrication du tabac.

Il est important que nos cultivateurs cultivent bien le tabac et le préparent avec attention afin de donner une bonne renommée à ce r roduit. Par ce moyen, ils s'assureront de larges profits. 



\section{QUELQUES NOTIONS}

SUR

LA CTLTLRE RT LA PREPARATION DU TaBaC.

Lorsque l'on cutive le tabac, il ne suffit pas de chercher a produire des plantes bien fournies, avec des feuilles graud s et larges, c'est.à-dire qu'il ne suffit pas d'oblenir une récolte abondante, il faut encore, et surtout, arriver à obterir un tabac de bonne qualité, dont l'arôme soit agréable, et cette circonstance dépend non seulement de l'espèce de tabac que l'on cultive, mais particulièrement de la inanière dont on le cultive, et des soins que l'on 'apporte depiris son ensemencement jusqu'à ce qu'il soit propre à la consommation; et ces soins ont rapport au choix et à la préparation de la graine, au chuix de la nat re et de l'exposition du terrain, aux engrais à employer, à la culture proprement dite, au séohage après la récolte et à la préparation défi. nitive.

D'abord, il faut que le terrain ait une bonne exposition, à l'abri des vents du nord et du nord est, qu'il 
soit en fente rers le sud, et il faut le choisir sur lesg côtes plutôt que sur les plateaux. Les terres sahleuses contenant une faible quantité d'argile et des rósdus de végétanx en décomposition, donnent un tabac léger, d'une donceur el d'un arôme remalquables. Les teres noires, grasses at les terres franches produisent un labac très fort qui est employé le plis souvent pour la préparation du taliae en poudie. .

Mais quels que soient les terrains dont vous disFosiez, vous pouvez toujours les annélinir par les labouis et 'es amendenents, et vous raprocher de la meilleure qualité de terre pour la culture de cette plante. Ainsi, sur les trires fortes, argileuses, vous transportere\% dı sable et de bons composts faits avec de la chaux et re-débris de végétaux. Li vons avez affaire à des suls légers, s.iblonieux, voi,sles mélangerez avec de la marne argileuse, et sur les terrains compactes calcaires, vous répandrez de l'argile et de la terre végétale pour former une bonne couche végétale.

Après avois fait choix d'un sol comvenable, il fant songer à lui procurer l'engrais néc ssaire.

Le tabac drmande une lerre substantie:le bicn chargie d'engrais, el les engrais les ptus actifs sont les nu illeus pour sa culture.

La terre doil êlre bien meuble, bien fiıfe, avec une surface plane qui alsorbo les eaux de pluies, mais qui retient son humidilé.

Pendant que vous vous occupez de la préparation 
de votre terrain, vous devez, au eommencement du mois de mai, préparer votre couche chaude et semer votre graine. La grandeur de votre couche dépendra de la quantité de tabac que vous voulez planter.

Autant que possible, vous emploierez de la semence de la dernière récolte, car la graine de tabac devient en vieil!issant plus lente à germer, et elle perd même la faculté de germer au bout de trois ou quatre ans. Il est toujours pruitent d'en essayer quelque temps d'avance un nombre déter iné de grannes dans une petite caisse, afin de se rendre compte de sa valeur et de voir si on peut l'employer sûrement.

Pour arriver à maturité, il fant généralement au tabac deux mois et demi à trois mois, depuis la transplantation jusqu'à la récolce. L'époque de la transplantation la plus favorable est du ler au 15 juin. Le tabac peut ainsi être récolté de la fin d'août au 15 septembre.

Lorsque vous voulez planter le tabac, choisissez autant que possible un temps couvert et humide, mais non un temps qui fait présager de fortes pluies ou un orage, car la violence de la pluie abîmerait les jeunes plantes.

Après sa transplantation le tabac exige des soins continuels. Il faut le prutéger contre le froid, contre un soleil trop ardent, et surtout contre les insectes.

Les arrosements sont très-importants. 
Si nous laissons la terre livrée à elle-même, à moins que le temps ne soit pluvieux, elle se desséchera bientôt autour des plantes dont les racines ne pourront trouver leur no: rriture faute d'humidité, et qui languiront et dépériront, ou mûriront prématurément. Nous ne vous dirons pas : "Arrosez tous les j.urs, tous les deux jours, tous les trous jours. " Il faut powvoir juger quand une plante a soif, ou plutôt, vous devez savoir deviner quand elle estisur le point d'avoir soif, car si vous attendez qu'elle l'ait, elle souffre, et toute plante qui souffre est retardée. Maintenez donc constamment un certain degré d'humidité dans la terre où les racines puisent les sucs nourriciers. "Il faut de l'arrosement, pas trop n'en faut." Les arrosements doivent toujours se faire au déclin du jour. Les arrosements exagérés et trop pronlongés auraient pour effet d'aciiver la végétation ninre mesure et de reta der la maturité du tabac. 1 orsque la plante a atteint tout son développemont, la tige se durcit, les feuilles rerouvrent la terre et empêchent le soleil d'absorber l'humidité qui se maintient dans le sol. Alors les arrosements deviennent inutiles et doivent cesser tout a fait

On emploie l'eau pure pour arroser. Cependant si l'nn jugeait que la culture languit faute d'engrais, il scrait bon d'y ajouter des urines ou du jus de fumier qui rendraient à la terre les principes ferti. lisants qui lui manqueraient.

Habituellement, et par un temps sec, on arrose tous les soirs pendant la première semaine, puis on 
diminue peu à peu les arrosements à mesure que la plante se développe.

L'opératión du sarclage doit être faite souvent afin de conserver le sol net.

Lorsque la plante a acquis un développement suffisant et qu'elle forme sa flèche, c'est-à dire que la tête se dispose à se séparer en bourgeons destinés à porter des fleurs et des graines, vous devez arrêter sa croissance en rompant cette tète ou flèche. Par ce moyen on permet aux feuilles de prendre toute leur extension. Un peu plus tard, il se forme entre la feuille et la tige des bourgeons qu'il est nécessaire d'enlever à mesure qu'ils se montrent, afin qu'ils ne s'emparent pas irutilement de la sève qui doit être entièrement réservée à la nourriture des feuilles, ce qui s'appelle vulgairement dragonner.

Comme toute la nourriture q"e les racines puisent dans la terre passe dans les feuilles, le tabac sera d'autant plus fort qu'on en laissera un plus petit nombre, lors de l'ététage, et au contraire il sera d'autant plus faible qu' ou en laissera plus. Ainsi, pour avoir du tabac fort, on laissera 10 à 12 feuilles; pour obtenir un tabac moyen, on en laissera 15 ou 16, et enfin, si l'on veut prodnire un tabac doux, on en laissera 20 et plus. On supprime donc toutes les autres, et on choisit pour les supprimer, toutes celles qui pourraient être avariées, ou qui présenteraient la moindre apparence. 


\section{RÉCOLIE DU TABAC.}

La maturité du tabac arrive de la fin d'août an 1 j septembre. Elle s'amnonce par l'aspect que prenucut les feuilles qui pâlisseut et qui, du vert vif et agréabie, tournent au jaune pâle et obscure tout à la fois Dis taches jatumes veruatres apparaiss. nt et sont surtout visibles lorsque l'on regarie les fetilles contre le soleil. D'un aucre côté, les feuilles semblent fatigures, elles ne se maintiennent p'us avec cette espèce de vigueur qu'elles montraient auparavaut pendant la croissance. Elles s'inclinent vers la terre, et leur surface parait se rider. Alors, il fatit couper le tabac a vant que la maturité soit trop prononcée. Le tabac coupé trop vert pèse moins quand il est sec, contient moins de sels, conserve toujours une mauvaise couleur et est de mauvaise qualitó.

Il faut ensuite le faire sécher, jusqu'à ce qu'il devienne janne ou brua. Alorson dêtache les feuilles du coton et on les pile.

Si l'on veut classer le tabac suivant la force, on se rappellera que les feuilles du pied donnent le tabac le plus fort. celles du milieu, un tabac moyen. et celles du sommet, un tabac doux. On mettra donc à part les fuuilles de ces trois espèces, en en comptant un quart pour la première, la moitié pour la seconde et le dernier quart pour la troisième.

Si vous ne cultivez du tabac que pour vous-même, laissez-le en pile ou pressé en caisse pendant sıx ou 


\section{$-11-$}

huit mois. Le tabac s'améliore beancoup en vieillis. sant dans cette position; i achève de former sa coulleur, perd son goût de jeune et acquiert tout l'arôme dort il est susceptible.

Si vous cuitivez le tabac pour le vendre aux fabri ques, il vous suffira de le préparer comme ci-dessus.

Mais pour le commerce vous aurez à vous conformer à la loi dı Revenu Intérieur, dont voici un résumé exact: 



\section{LE TABAC CANADIEN}

$\mathrm{ET}$

LOIS QUI EN REGISSENT LA CULTURE ET LA VENTE.

Q.- Quelle est la loi q i régit la culture et ia vente du tabac canadien?

R.-C'est l'Acle pour refondre et amender les actes concernant le Revenu de l'Interieur, chapitre 19 des Staluts du Canada, sanctionné le 7 mai 1830.

$\mathrm{Q}$-Quelles défintions cette loi donne t-elle du tabac et des fabricants de tabac?

R.-lo Tabac brul signifie tout tabac non fabriqué, ou les feuilles et tiges de la plante avant d'avoir subi aucun procédé de fabrication ;

Tabac fabriqué signifie et comprend tout article fait avec du tabac brut par quelque procédé que ce soit.

Le tabac étalon de toute sorte est celui qui est composé de dix pour cent d'eau et de quatre-vingt. dix pour cent de matière solide, et le poids de tout tabac sera calculé et porté dans tous les comptes, états et rapports, d'après cet étalon : 
Manufacluie de labuc signifie et comprend tout lit u ou établissement dans lerjucl lo: tabac est fabriqué ou mis en cenvere ;-et tous ateliers, bureaux, magasins, ent?'pôts, boutiques, appentis, cour ou autre flare où la matière première est ou dot être emmagasiné, on lans lu-i "ls se foursuit ou doit se pou:suivre tout procédé du ressu t de la fabrication en préparation du tabac, ou dans lesquels les prodiuits de 'a manufacture sont ou doivent être emmagasinés-sernut reputés compris dans la manufacture de tabac à laquelie ils sont altachés ou dont ils déper dent, et en former partie;

Fabricant de tabac signifie et comprend toute perso: ne qui, par elle-même ou son agent, f.brique ou met eu œuvra ou prépare de quelque manière que ce soit le tabar b: ut en tabac à fumer, à chiquer ou à priser, ou eu toute autre espèce; et la fabrication ou préparation des cigares constituera la fabrication du tabac dans le sens du présent acte.

\section{DES LICENCES.}

Q.-Faut-il une licence pour cultiver du tabac pour le sommerce?

R.-Oui il faut une licence pour avcir le droit de cultiver du tabac pour le commerce; mais il n'en faut pas si on "cultive du tabac sur ses terres ou 
propriétés et si on le fabrique uniquement pour son usage et celui des membres de sa famille qui demeu. rent avec soi sur la terre ou propriété où le tabac est cultivé, et non pour le vendre, et le tabac ainsi fabriqué ne sera non plus sujet au droit d'excise; pourvu toujours que la q antité ainsi cultivée et fabriquée en une même année n'excède pas trente livres pour chaque membre alulce de la famille du sexe masculin, demeurant su la terre comme susdi:.

Q.-A qui fau! il s'adresser pour oblenir cetle licence?

$\mathrm{R}$ - - A u percepteur du revenu de l'Intérieur, ou à tout autre officier nommé à cette fin, dans le dis. trict ou la division du revenu ou l'on veut enltiver ou fal riquer le tabac.

Les officiers è revonu de l'Intérieur dont les noms suivent sont autorisés à délivrer ces licences.

James Gow ............Percepteur.

Ont. C. Dunlop ...............Sous-percepteur...Chatham, do John Spence..............Percepteur..........Londen, do W. Gill....................Sous-percepteur...St. Thomas, do M. Battle.................Percepteur.......... Ottawa, do P. MeGlashan............. do …......Sarnia, do M. M. Mulhern.......... do …......Cornwall, do J. L. Vincent ............ do ..........Montréal, Que. Geo. Larue............... do .........Québec, do E. McEachern........... do .......... Ormstown, do David Desroches......... do o........Ste. Thérèse, do R. M. Leprohon........... do ........... Joliette, do Aimé Roy ................. do do Pierre Reynier........... do Sorel, do do 
C. A. Boivin..............Percepteur.........S. Hyacinthe, Que. John Griffith............ do ..........Sherbrooke, do C. D. Hebert............ do ........ Trois-Rivières, do W. Dibblee ...............Sous-percepteur.. Woodstock, N. B. J. T. Griffin .............Pcrcepteur........Chatham, N. B.

Q.-Comment doit-être faite cetle demande?

R. - Chaque demande de licence indiquera exactement le nom du requérant, le lieu où il réside et veut cultiver le tabac pour le commerce, et spérifiera le nombre maximum de plants, et le maximum de l'étendue de terrain que le requérant se propose de mettre en culture durant l'année pour laquelle il demandera cette licence.

Q.-A quelles conditions cette licerce pour cultiver du tabac pour le commerce est-elle accordée?

R.-Une licence autorisant la culture du tabac pour le commerce, sur l'échelle mentionnée dans la demande de lice ice, pourra être accordée gratuitement, à condition que ía personne à qui elle sera accordée s'enga a à faire des rapports exacts et fidèles de la quantité de tabac qu'elle aura cultivé pour le commerce, et des noms et domiciles des marchands de tabac licenciés ou autres personnes à qui ce tabac sera vendu, ou comment il en sera autrement disposé.

$\mathrm{Q}-\mathrm{Quand}$ devra être renouvelée rette licence?

R.-Tous les ans, après le 30 de juin. c'est-à-dire qu'il faudra la demander en mai et en uin pour l'année courante. 
Q.-Faut-if une licence pour fabrique! du tahac. canadien pour le commerce?

R-Oui, il faut une licence que l'on obtient du percepteur du Revenu.

Q.-Cette licence est elle accordée gratuitement?

$\mathrm{R}$-Non, la personne en faveur de qui il sera accordé une licesce pour la fabrication de tabac cultivé en Canada, exclusivement, devra, en recevant cette licence, payer au percepteur du Revenu de l'Intérieur la somme de cinquante piastres.

Si l'on fabrique exclusivement du tabac étranger, la licence coûtera soixante-quinze piastres.

Q.-Quelles sont les conditions imposées pour obtenir cette licence?

R.-Une licence de fabricant de tabac pourra s̀tre accordée à toute personne qui se sera conformée aux dispositions de la loi, pourvu que la concession à cette licence ait été approuvée par l'inspecteur du district et que le requérant ait, conjointement et solidairement avec pas moins de deux ni plus de six cautions solvables, consenti une obligation à Sa Majesté, ses héritiers et successeurs, pour une somme égale au montant auquel le percepteur du revenu de l'Intérieur estimera les droits que doit payer la personne à qui la licence est accordée, pendant deux mois de la durée de la licence.

Q.-Que doit contenir la demande de licence pour la fabrication du tahas? 
R.--Chaque demande de licence porar la fabication du taljac contiendra une liste et description des outils et machines employés ou que l'on se proposera d'employer dans l'opération pour laquelle !a licence sera demandée, et spécialement de toutes presses, machines et moulins à transher, en indiquant la partie de la bâtisse dans laquelde ils seront employés. Ello devra mentionner aussi si du tabac brut ou en feuille étranger ou importé doit êtré employé ou introduit dans la manufacture à l'égard de laguelle la licence est demandéê.

\section{DROITS D'EXCISE OU TAXES.}

Q.-Quels soni les droits d'excise ou taxes qu'il fant payer pour vendre du tabac?

R.-Sur le tabac fabriqué et le tabac à priser de toute espèce (excepté les cigares) fait en tout ou en partie de tabac en feuille étranger ou importé et contenant pas moins de dix pour cent d'eau, au poids, el ainsi dans la même proportion pour loule quantité d'eau plus ou moins grande, sur chaque livre on yuantité moindre qu'une livıe, vingt centins.

Sur les cigares faits de tabac en feuille étranger o!ı importé, pour chaque livre, quarante centins.

Sur le tiabac fabriqué de toute espèce (excepté les 
cigares et le tabac cantadien en tarquelte ordiraire) lorsqu'il est fait uniqueuent de tabac ou crû dü Canada et dans une manufacture où il n'est employé ou garder aucun tabac importé ou étranger, et con tenant pas moins de dix pour cent d'eau, au poids, et ainsi dans la mème proportion pour toute quantité d'eau plus ou moins grande, sur chaque livre ou quantité moindre qu' une livre, quatorze centins.

Sur les cigares faits exclusivement de tabac cultivé au Canada, pour chaque livre, trente centins.

Sur le tabac canadien, autrement appelé tabac blanc. en torquette, étant la feuille non pressée, ma:s roulée et faite entièrement de tabac brut de la provenance du Canada, pour chaque livre ou quantité moindre qu' une livre, quatre centins.

Q.-Le cultivateur peut-il vendre le tabac en feuille au détail ?

R. -Non; il peut vendre le tabac en feuille au manufacturier seulement ou au fabricant de tabac, ou au marchand licencié. Dans ce cas, il ne paie aucun droit d'excise, pourvu qu'il se conforme aux exigences de sa licence qui lui permet de cultiver du tabac pour le commerce.

Q.-Mais comment peut-il vendre son tabac?

K.-Il peut le vendre en le préparant en torquette ordinaire ou en rouleaux ne pesant pas plus de deux livres chaque, et chaque rouleau sera attaché au moyen de ficelles ou autrement, et enveloppé dans une bande de papier ou autre bande ou enveloppe, afin que l'estampille puisse y être apposée. 
Q.-Pour fabriquer ainsi du tabac en torquette ordinaire ou en rouleaux, le cultivatetir a t-il quelque droit à payer?

R.-Tout cultivateur de labac licencié qui désire manufacturer le tabac ou partie du tabac cultivé sur sa ferme en tabac canadien en torque'te ordinaire, pour le commerce, peut le faire sans payer aucun droit de licence, pourvu qu'il ait, en demandant sá licence, déclaré son intention de faire cette fabrication et que la licence qui lui sera accordée runferme une autorisation à cet effet.

Mais il devra estampiller le tabac ainsi manufacturé, avant de le sortir de la ferme pour le vendre, c'est-à-dire payer le droit de quatre centins par livre.

Q.-Où le cuitivateur achètera-t-il ces estampilles ?

B.-Chez le percepteur du Revenu de l'Intérieur de qui il a obtenu sa licence ou de toute autre personne que le gouvernement aura nommé pour cet ohjet.

Q.-Si le cultivateur ne se conforme pas à la loi, à quoi s' expose-t il ?

R.-Il s'expose à payer une amende de cinquante piastres pour la première offense, et pour toute récidive, une amende de deux cents piastres, et, en oatre, à voir tout son tabac confiequé. Bien plus, il encourra et paiera de plus que les amendes, deux fois le montant du droit d'accise et de licence qu'il aurait dù payer en ve:tu de la loi. 


\section{AMENDES.}

Toute personne non licenciée qui a en sa possession une presse à tabac, moulin pour le hacher ou le moudre ou toutes autres machines destınées à la fabrication du tabac, est passible d'une amende de $\$ 50.00$ à $\$ 1 \mathrm{C} 0.00$.

Toute personne r:on licenciée en la possession de laquelle on trouve du tabac brut ou manufacturé, délié ou paqueté, etc., est passible d'une amende de $\$ 50$ à $\$ 200.00$.

Quiconque résiste aux officiers du revenu ou se porte sur eux à des voies de fait est coupable de félonie et puni, s'il est déclaré coupable, par emprisonnement pour une période de six mois à cinq ans. 








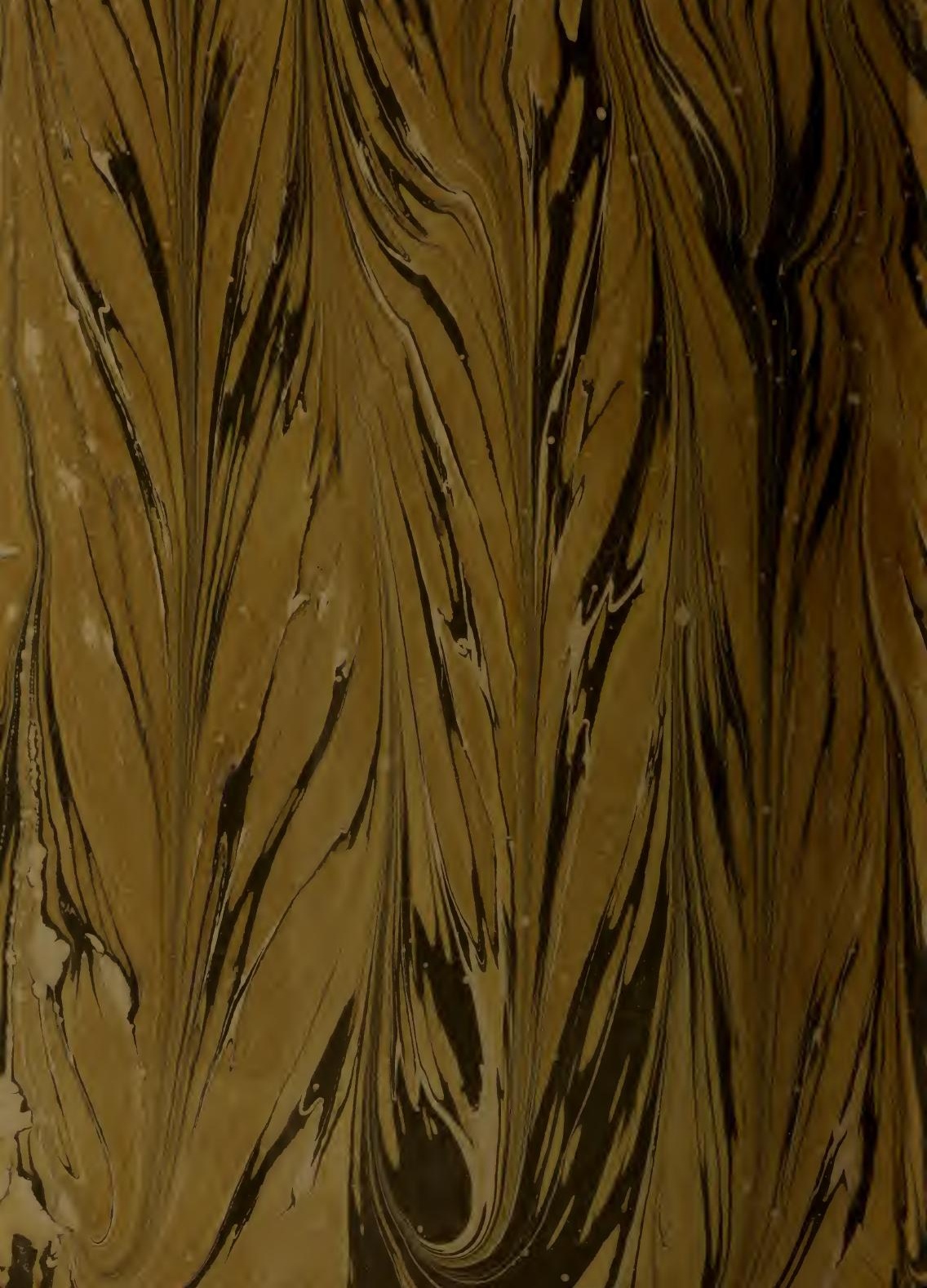

